\title{
Sustainable synthesis of Co NPs@Graphited carbon microspheres as an efficient electrocatalyst for the oxygen-evolution reaction
}

\author{
Hong-Qiang Wang ${ }^{\mathrm{a}}$, Dong-Cai Zhang ${ }^{\mathrm{a}}$, Xiao-Hui Zhang ${ }^{\mathrm{a}}$, Ze-Sheng Li ${ }^{\mathrm{b}}{ }^{\text {* }}$, Guan-Hua \\ Yang ${ }^{\mathrm{a}}$, Yong-Sheng $\mathrm{Wu}^{\mathrm{a}}$, Jing-Jing $\mathrm{Ji}^{\mathrm{c}}$ and Qing-Yu $\mathrm{Li}^{\mathrm{a}}{ }^{\mathrm{*}}{ }^{\mathrm{a}}$ \\ a Guangxi Key Laboratory of Low Carbon Energy Materials, School of Chemistry and pharmaceutical Sciences, \\ Guangxi Normal University, Guilin, 541004, China. \\ b Development Center of Technology for Petr ${ }^{\circ}$ Chemical Pollution Control and Cleaner Production of Guangdong \\ University, College of Chemical Engineering, Guangdong University of Petr ${ }^{\circ} \mathrm{Chemical}$ Technology, Maoming, \\ Guangdong, 525000, China. \\ c Ningbo Institute of Materials Technology and Engineering, Chinese Academy of Science, Ningbo, Zhejiang, \\ 315000, China. \\ Correspongding authors \\ E-mail addresses \\ *Qing-Yu Li, lingqingyu62@126.com Fax: +86-0773-5858562 \\ *Ze-Sheng Li, 1zs212@163.com
}

\begin{abstract}
A temperature-controlled water-swelling and catalytic graphitization associated strategy has been demonstrated for the sustainable synthesis of perfect Co nanoparticles-wrapped graphited carbon microspheres (Co@GCMs) from a green precursor of corn starch at a relatively low temperature. This strategy results in a relatively high degree of graphitization for GCMs at $900{ }^{\circ} \mathrm{C}$ and excellent microsphere structure succeeded from the precursor. The Co nanoparticles were well enwrapped by porous graphited carbon nanofilms which have been grown on the surface of GCMs. Such a unique Co@GCMs hybrid architecture could afford a very high catalytic activity as well as a desirable electrochemical stability for the oxygen evolution reaction (OER). The Co@GCMs sample sintered at $900{ }^{\circ} \mathrm{C}$ shows an ideal onset potentials of $1.36 \mathrm{~V}$ and a very high catalytic current density of $6.43 \mathrm{~A} \mathrm{~g}^{-1}$ at $1.60 \mathrm{~V}$ potential, which demonstrates a positive application prospect of this hybrid material.
\end{abstract}

Keywords: OER; Electrocatalyst; Porous carbon microspheres; Starch

\section{Introduction}

Since the discoveries of fullerenes in 1985 and carbon nanotubes in 1991, in the world many scientists have carried out comprehensive researches on carbon materials $[1,2]$. According to different methods and carbon precursors, diverse morphologies of carbon materials such as conical carbon, micro-roll carbon, micro-sphere carbon, onion-type carbon and flake-type carbon have been successfully prepared [3]. Micro-sphere carbon is considered as a kind of promising carbon material for the application of numerous technical fields, due to its outstanding physicochemical properties and favorable geometrical structures [4]. Mesocarbon microbead is a typical micro-sphere carbon material which was evolved from the pitch-based compound during heat treatment in the early 1970s [5]. This micro-sphere carbon shows many excellent properties, such as well self-sintering performance, excellent electrical or thermal conductivity and high packing density, etc [6]. Therefore, it has been widely used 
as a high-density and high-strength carbon material, lithium-ion secondary battery anode material, catalyst carrier and high-performance chromatographic column filler [7-9]. In recent years, many non-pitch precursors were used to construct various solid, porous, hollow, even polyhedral carbon microspheres, which greatly enriched the application of micro-sphere carbon materials in related fields [10-12]. Nowadays, with the severe shortage of energy, the increased awareness of environment protection and the requirements of sustainable development strategy, environmentally friendly raw materials and low-energy-consumption preparation technology are highly essential to sustainable production of high-quality and cost-effective functional carbon materials [13].

Obviously, incorporating sustainable concepts into the synthesis of micro-sphere carbon materials would be a meaningful research task. Polysaccharide biomass is a quite suitable choice for the sustainable synthesis of carbon materials, because of its low price, huge amount, rapid regeneration, as well as environmental friendship [14, 15]. Among the numerous polysaccharide, starch is a natural polymer and can be obtained from various renewable plant resources $[16,17]$. Besides, starch has a typical spheroidal morphology and appropriate micron scale, which is a promising carbon source for the preparation of micro-sphere carbon materials. For example, Wang et al. have reported the synthesis amorphous carbon microspheres from potato starch, by a low-temperature stabilization and high-temperature carbonization two-step process [18]. Liu et al. also reported that sulfur-doped amorphous carbon microspheres can be synthesized on a large scale by hydrothermal carbonization of sulfur and starch mixed solution [19]. Yu et al. recently demonstrated the synthesis of amorphous carbon-encapsulated metals microspheres by heating carbonization process with maize-derived starch as carbon source and metal nitrate as metal precursors [20]. All these works presented that, as one of the renewable resources, starch is a promising precursor for production of a variety of functional micro-sphere carbon materials.

Currently, starch-based carbon microspheres have been studied as electrode materials in diversified electrochemical energy storage and conversion, e.g. lithium-ion battery, supercapacitors and water electrolysis [21-23]. A high degree of graphitization along with a good electrical conductivity for the carbon materials is highly desirable for the sake of excellent electrochemical properties [24, 25]. However, most of the reported starch-based carbon microspheres are of amorphous carbon texture [16-25] and there is no available example concentrated on graphited carbon existence. Because of their relatively low electrical conductivity, the application of these carbon microspheres is limited to some extent, so the degree of the graphitization for these starch-based carbon materials becomes a key element for their functionality. On the other hand, developing energy-saving technology for the synthesis of graphited carbon materials is the necessary way to make it from basic research to practical application. Actually, graphited carbon materials can be prepared by traditional high-temperature solid-phase sintering method, in which a very high temperature of $2000 \sim 3000{ }^{\circ} \mathrm{C}$ is required and the energy consumption is extremely considerable [26]. Fortunately, the recently developed low-temperature graphitization technology promoted by catalysis of transition metals ( $\mathrm{Fe}, \mathrm{Co}, \mathrm{Ni}$, etc.) gives ones great encouragement by the fact that this technology can realized graphitization of biomass below $1000{ }^{\circ} \mathrm{C}$ [27]. More recently, Lei 
and co-workers accomplished the preparation of graphited carbon fragments with starch gelatinization as raw material and $\mathrm{FeCl}_{3}$ as catalytic precursor [28]. However, the controlled synthesis of graphited carbon microspheres with integrated globular structures from starch microspheres still remains a challenge.

Starch is a kind of polycrystalline polymer, which is composed of amylose and amylopectin molecules, and forms the starch granule. Usually, starch granules can be dissolved in hot water $\left(>75^{\circ} \mathrm{C}\right)$, with the crystalline structures of amylose and amylopectin molecules is lost [29]. This means that the globular structures of starch are destroyed irrevocably. Therefore, it is no longer feasible to construct globular carbon materials from the resulted starch gelatinization indicated by previous work [28]. On the other hand, from the catalytic graphitization point of view, their homogeneous mixing of carbon sources and precursors of catalytic medium is very important. By all accounts, two prerequisites for construction of graphited carbon microspheres from starch granules become apparent: (i) the globular structures of starch must be maintained in the heat treatment process, and (ii) the catalytic precursors should be uniformly distributed onto and into the starch granules. In most cases, the two conditions belong to a pair of contradictions that caused a great barrier for the design and synthesis of starch-based graphited carbon microspheres.

In this investigation, we demonstrate the controlled synthesis of the cobalt (Co) nanoparticles (NPs)@ Graphited carbon microspheres (GCMs) from a sustainable precursor of corn starch, at a relatively low temperature of $900{ }^{\circ} \mathrm{C}$ by Co-metal catalytic graphitization. Expressly, a temperature-controled water-swelling strategy was first introduced for obtaining desirable dispersion of Co precursor into the inside of starch, while avoiding the dissolution and collapse of starch microspheres. The as-prepared Co@GCMs sample has several obvious features structurally: (i) excellent microsphere structure is succeeded from the precursor of starch, (ii) the Co NPs were well enwrapped by porous graphited carbon nanofilms, (iii) a relatively high degree of graphitization for GCMs is achieved. Furthermore, the newly designed Co@GCMs composite structure showed a very high activity for electrocatalytic oxygen evolution from the Water.

\section{Experimental}

\section{Synthesis of materials}

In a typical synthesis of Co@ GCMs samples, 0.54g cobalt acetate $\left(\mathrm{Co}\left(\mathrm{CH}_{3} \mathrm{COO}\right)_{2} \cdot 4 \mathrm{H}_{2} \mathrm{O}\right)$, $3 \mathrm{~g}$ corn starch (amylose content, $\sim 25 \%$ ) and $5 \mathrm{ml}$ of deionized water were mixed in a beaker, then the sample in the beaker was stood to heat for 40 minutes in the $65{ }^{\circ} \mathrm{C}$ constant temperature water bath. Next, the sample was dried at $40{ }^{\circ} \mathrm{C}$ for four hours in the electric oven thermostat blast to obtain the complexed precursor of starch and cobalt acetate (cobalt acetate-treated starch). Four parts of the precursors were further heated to $400{ }^{\circ} \mathrm{C}, 600{ }^{\circ} \mathrm{C}, 800$ ${ }^{\circ} \mathrm{C}$ and $900{ }^{\circ} \mathrm{C}$, respectively, from room temperature at the heating-up rate of $2{ }^{\circ} \mathrm{C} /$ min under nitrogen flow, then held for 3 hours, and finally cooled to room temperature naturally. Finally, four samples were obtained with the four different sintering temperatures. In addition, for the of purpose comparison, starch-derived carbon (C) was also synthesized by direct sintering at $900{ }^{\circ} \mathrm{C}$ without the cobalt acetate.

\section{Characterization of materials}


The X-ray diffraction (XRD) measurement was carried out with a Bluker D8-Advance diffractometer with $\mathrm{Cu} \mathrm{K} 1$ radiation $(\lambda=1.5406 \AA)$. The data was collected between $10^{\circ}$ and $90^{\circ}$ at a step size of $0.020^{\circ}$. Raman spectra were obtained in the frequency range of $500 \sim 3500 \mathrm{~cm}^{-1}$ with a laser Raman spectroscope (invia, Renishaw). The morphologies of the samples were examined by using a field emission scanning electron microscopy (SEM, Philips, FEI Quanta 200FEG) and a transmission electron microscopy (JEOL-2010). The specific surface area and pore structures of the samples were investigated using physical adsorption of nitrogen at the liquid-nitrogen temperature $(77 \mathrm{~K})$ on an automatic volumetric sorption analyzer (SA3100 Beckman Coulter, USA).

\section{Electrode preparation and electr ${ }^{\circ} \mathrm{Chemical}$ measurements}

The catalyst samples and binder polyvinylidene fluoride (PVDF) were dispersed in $\mathrm{N}$-methyl-2-pyrrolidone (NMP) with the weight ratios of two to one. After the mixture is stirred evenly for 2 hours, the catalyst ink was deposited on the surface of on the spectrum of pure graphite electrodes with a geometric area of $0.28 \mathrm{~cm}^{2}$ and dried at $80{ }^{\circ} \mathrm{C}$ for $1 \mathrm{~h}$. The electrocatalyst loading on the spectrum of pure graphite electrodes was normally controlled at $0.1 \mathrm{mg} \cdot \mathrm{cm}^{-2}$. The experiments were carried out at $25{ }^{\circ} \mathrm{C}$ controlled by a water-bath thermostat. All electrochemical measurements were carried our in $0.1 \mathrm{M} \mathrm{KOH}$ aqueous solution with a three-electrode cell on the IM6 electrochemical workstation (Germany). A platinum (Pt) foil and reversible hydrogen electrode (RHE) were used as the counter and reference electrodes. The Cyclic Voltammetry (CV), linear-sweep voltammograms and electrochemical impedance spectroscopy (ESI) techniques were applied to investigate the catalytic performances on oxygen evolution reaction. The Chronoamperometric technique was selected to evaluate the electrochemical stability of the as-prepared catalyst samples.

\section{Results and discussion}

Fig. 1 displays the typical field emission scanning electron microscopy (FE-SEM) images of Co@GCMs sample obtained by sintering at $900{ }^{\circ} \mathrm{C}$. It is very obvious that the sample has a similar spheroidal structure with diameter of $5 \sim 10 \mu \mathrm{m}$ depicted in Fig. 1 A. A higher-magnification SEM image (Fig. 1 B) shows that the carbon microspheres have an accidented surface structure with innumerable Co NPs grown on it (the Co phase is confirmed by XRD hereunder). Partially enlarged SEM image (Fig. 1 C) further demonstrates that the surface structure of GCMs is made up of highly crumpled carbon nanofilms in a large area. More importantly, these carbon nanofilms display a translucent feature and the Co NPs were well enwrapped by them, as can be seen from Fig. 1 D. The particle size of these Co NPs varies from 50 to $150 \mathrm{~nm}$ by the SEM measurement. To further insight into the internal structures, the sectional SEM images of Co@GCMs are presented in Fig. 2 A and B. It can be clearly seen that the Co NPs are imbedded the inner structure of GCMs (see the arrow), and the thickness (about $20 \mathrm{~nm}$ ) of carbon nanofilms is also observed from outer section structure. Furthermore, some significant nanopores (see the circles) on the carbon nanofilms are discovered from Fig. $2 \mathrm{C}$ and $\mathrm{D}$, which might play an important role in communicating the internal Co NPs to external environment.

Representative TEM images of Co@GCMs are presented in Fig. 3, which reveal the detailed porous structures and graphited structures of the above-mentioned carbon nanofilms. 
A lower-magnification image (Fig. 3 A) exhibits a number of Co NPs are well contacted with the carbon nanofilms. It is noteworthy that individual Co NPs have penetrated the carbon nanofilms and corresponding nanopores (about $40 \mathrm{~nm}$ denoted by arrow) are generated as shown in Fig. 3 B. This result is well consistent with the findings of SEM observation from Fig. 2 D. More interestingly, a great deal of smaller nanopores $(3 \sim 5 \mathrm{~nm})$ are homogeneously dispersed on the carbon nanofilms everywhere, which may be obtained from the smaller Co NPs at early lower-temperature stage. Expressly, the high-resolution TEM images (Fig. 3 C and D) show the expected graphited structures of the carbon nanofilms with visible graphite planes $\left(\mathrm{d}_{002}=0.34 \mathrm{~nm}\right)$.

On the other hand, nitrogen adsorption-desorption analysis of the Co@GCMs sample (Fig. 4 A) exhibites the typical characteristics of mesoporous materials, with increasing adsorption volume as well as pronounced desorption hysteresis loop (with a BET surface area of $280 \mathrm{~m}^{2}$ $\mathrm{g}^{-1}$ ). The corresponding pore size distribution (Fig. 4 B) indicates that the sample has a abundant small-mesoporous texture $(3 \sim 5 \mathrm{~nm})$ and partial large-mesoporous texture (30 50 $\mathrm{nm})$. The properties of mesoporous structures are in accord with the results of TEM observation.

In order to demonstrate the formation mechanism of Co@GCMs from precursor of starch, XRD patterns and Raman spectrums of the samples sintered at different temperatures are showed in Fig. 5. The samples sintered at $400{ }^{\circ} \mathrm{C}, 600{ }^{\circ} \mathrm{C}, 800{ }^{\circ} \mathrm{C}$ and $900{ }^{\circ} \mathrm{C}$ exhibit homologous characteristic diffraction peaks of Co metal at $2 \theta=41.2^{\circ}, 51.5^{\circ}$ and $75.8^{\circ}$ from XRD patterns (Fig. 5 A). The intensity of the diffraction peaks becomes stronger and sharper with the increase of temperature, indicating the size of Co NPs increases gradually with the increasing temperature. It is known that the 002 diffraction peak of carbon materials derives from the diffraction of the laminated structure of graphite. The sample sintered at $400{ }^{\circ} \mathrm{C}$ only shows a broad diffraction peak with stronger intensity at $2 \theta=23^{\circ}$, which indicates that the sample is in the form of amorphous carbon substantially. But for the sample sintered at 600 ${ }^{\circ} \mathrm{C}$, the broad diffraction peak at $2 \theta=23^{\circ}$ becomes weaker, largely because of its more orderly graphite crystallites arranged in a two-dimensional direction. When the temperature increases to $800{ }^{\circ} \mathrm{C}$, the sample reveals a clear characteristic peak (002) of graphite with moderate intensity at $2 \theta=26.2^{\circ}\left(\mathrm{d}_{002}=0.3398\right)$, demonstrating that the graphite crystallites become graphite crystals with large two-dimensional size, namely the graphited carbon is formed at this point. Remarkably, for the sample sintered at $900{ }^{\circ} \mathrm{C}$, its characteristic peak (002) of graphite becames stronger and sharper $\left(\mathrm{d}_{002}=0.3378\right)$, which proves that Co-metal catalytic graphitization at this temperature has a ideal effect, with respect to its larger graphite crystal size, smaller layer spacing and the improved degree of graphitization. The degree of graphitization can be calculated up to $48 \%$ and $72 \%$ for the samples sintered at $800{ }^{\circ} \mathrm{C}$ and $900{ }^{\circ} \mathrm{C}$, according to the Maire and Mering formula [30]:

$$
G=\frac{0.344-d_{002}}{0.344-0.3354} \times 100 \%
$$

where $\mathrm{G}$ is the degree of graphitization of carbon materials, 0.344 is the interlayer spacing of non-graphited carbon, 0.3354 is the interlayer spacing of ideal graphited carbon. Usually, the bigger $\mathrm{G}$, the higher degree of the graphite, the larger size of the graphite layer and the 
less lattice defect are symbolized. The sample sintered at $900{ }^{\circ} \mathrm{C}$ with Co catalysis has a relatively high degree of graphitization, being close to that of high temperature graphitization at $2300{ }^{\circ} \mathrm{C}$ without any catalyst [31]. Thus it can be seen that the Co catalytic graphitization is a high-efficiency and energy-saving strategy for the synthesis of starch-based graphited carbon materials.

The Raman spectrums (Fig. 5 B) show the coincident carbon structures for the samples as indicated by XRD patterns. The sample sintered at $400{ }^{\circ} \mathrm{C}$ shows a couple overlapped broad peaks at $1386 \mathrm{~cm}^{-1}$ (band D) and $1596 \mathrm{~cm}^{-1}$ (band G) [32], as well as a dispersive peak from 2000 to $3000 \mathrm{~cm}^{-1}$, which indicate that the crystalline structure of the sample is relatively low, and the residual $\mathrm{sp}^{3}$ carbon is very extensive. The sample sintered at $600{ }^{\circ} \mathrm{C}$ still shows the overlapped broad peaks at $1343 \mathrm{~cm}^{-1}$ and $1593 \mathrm{~cm}^{-1}$, but the peak wide become narrow and there is no dispersive peak from 2000 to $3000 \mathrm{~cm}^{-1}$, indicating the residual $\mathrm{sp}^{3}$ carbon is reduced and crystalline structure is increased. For the sample sintered at $800{ }^{\circ} \mathrm{C}$, the two peaks at $1348 \mathrm{~cm}^{-1}$ (band D) and $1584 \mathrm{~cm}^{-1}$ (band G) is narrow and sharp, and a better separation of them is achieved. The intensity ratio of $\mathrm{D}$ to $\mathrm{G}$ peak $\left(\mathrm{I}_{\mathrm{D} / \mathrm{G}}\right)$ is 0.77 , which indicates that the sample is already graphite, but the degree of graphitization is not very high. In addition, a additional sharp peak is appeared at $2700 \mathrm{~cm}^{-1}$ ( $\mathrm{G}^{\prime}$ band), and the intensity ratio of $\mathrm{G}^{\prime}$ to $\mathrm{G}$ peak $\left(\mathrm{I}_{\mathrm{G}^{\prime} / \mathrm{G}}\right)$ is 1.65 , suggesting the sample is of a multilayer graphite structure [33]. For the sample sintered at $900{ }^{\circ} \mathrm{C}$, the $\mathrm{I}_{\mathrm{D} / \mathrm{G}}$ is 0.67 that is lower than that of sample sintered at $800{ }^{\circ} \mathrm{C}$, implying the higher degree of graphitization. At the same time, the broadband intensity at $1500 \mathrm{~cm}^{-1}$ is weaker (closer to baseline), which demonstrates that the residual $\mathrm{sp}^{3}$ carbon of this sample is very low. Particularly, the $\mathrm{I}_{\mathrm{G}^{\prime} / \mathrm{G}}$ of the sample sintered at $900{ }^{\circ} \mathrm{C}$ is 1.21 , which is closer to the value of graphene $\left(\mathrm{I}_{\mathrm{G}^{\prime} / \mathrm{G}=1}\right)$, suggesting the sample has a few-layer graphite structure [33].

The SEM images of the samples before sintering and sintered at different temperatures are further presented in Fig. 6. The sample of cobalt acetate-treated starch (before sintering) shows that their surface is extremely smooth and there are no obvious particles on it (Fig. 6 A). Meanwhile, the XRD pattern of cobalt acetate-treated starch (before sintering) indicates that a cobalt acetate hydroxide hydrate is formed (Fig. S1). Hence, it can be inferred that the cobalt acetate species has penetrated into the internal structure of starch microspheres (Fig. S2) during the hydration. On the other hand, through the comparison of SEM image for samples sintered at different temperatures (Fig. 6 B D), one can easily find the growth process of the graphited carbon nanofilms of Co@GCMs. Evidently, the crumpled morphology of graphited carbon nanofilms are emerged at $400{ }^{\circ} \mathrm{C}$, and their three-dimensional structure become remarkable at $600{ }^{\circ} \mathrm{C}$. However, the Co NPs are not yet appeared on graphited carbon nanofilms at this temperature. The sample sintered at $800{ }^{\circ} \mathrm{C}$ displays the similar three-dimensional crumpled nanofilms as the sample sintered at $600{ }^{\circ} \mathrm{C}$. Significantly, a great deal of Co NPs with smaller diameter have been grown on the graphited carbon nanofilms (Fig. $6 \mathrm{E} \sim \mathrm{F}$ ). Compared with sample sintered at $900{ }^{\circ} \mathrm{C}$, the graphited carbon nanofilms of sample sintered at $800{ }^{\circ} \mathrm{C}$ is thicker and there are no visible nanopores on it. Furthermore, it should be noted that the starch-derived $\mathrm{C}$ sintered at $900{ }^{\circ} \mathrm{C}$ at the absence of cobalt acetate is of a fragmentary microflakes structure (Fig. S3). Therefore, it is can be 
drawn the conclusion that the Co catalysis not only played a pivotal role in promoting graphitization but also made a significant contribution to the preserving of globular structures.

To better discuss the growth mechanism of the Co@GCMs evolved from precursor of starch, the crystalline structures of starch, temperature-controled water-swelling process as well as the multi-step sintering effects are schematically shown in Fig. 7. Commonly, the amylose and amylopectin molecules of starch overlap each other into bundle-like crystalline lamellae and left a lot of nano-voids beween them to form amorphous lamellae. The crystalline lamellae are often dense and can not be attacked by water molecules at room temperature. However, when the temperature is higher than its glass transition temperature of the starch $\left(60 \sim 70{ }^{\circ} \mathrm{C}\right)$ but below its pasting temperature $\left(75 \sim 85{ }^{\circ} \mathrm{C}\right)$, water molecules can easily get into crystalline regions of starch grains and the starch was rapidly swelled due to the separating of crystalline bundles [34]. In the present case, a suitable swelling temperature of $65{ }^{\circ} \mathrm{C}$ is selected, thus the water molecules can evenly infiltrate into the starch grains. Meanwhile, the cobalt acetate hydroxide hydrate can be also evenly distributed into the internal structure of starch. Importantly, the cobalt acetate-treated starch is able to maintain a good globular structure after the water-swelling process. During the sintering process, the Co metals have been formed within the carbon microspheres at a low temperature of $400{ }^{\circ} \mathrm{C}$. Due to the uniform distribution of Co metal in carbon microspheres, efficient catalytic carbonation at $400{ }^{\circ} \mathrm{C}$ is readily achieved that provides a protective screen for the desirable globular structures in spite of concave surface. At this stage, the amorphous carbon and the Co metal are close contacted each other, which paves the way for the next catalytic graphitization. At the stage of $600{ }^{\circ} \mathrm{C}$, the catalytic effect of Co metal promotes combination of conplane crystallites gradually into larger crystalplane body, while the carbon grid plane is not orderly arranged in the direction of the vertical plane (namely the graphite crystals have not formed). In addition, the closely stacking of crystallites causes a more obvious concave surface for the carbon microspheres. When the temperature is further raised to 800 and $900{ }^{\circ} \mathrm{C}$, the catalytic effect of Co metal can promote rearrangement of random crystalplanes into ordered graphite crystals. Finally, crumpled graphited carbon nanofilms are grown on the surface of graphited carbon microspheres.

The development of an efficient catalyst for the oxidation of water to oxygen, namely oxygen evolution reaction (OER), is of great importance in the sustainable fuel production systems, because it provides available source of electrons for hydrogen production or the direct conversion of carbon dioxide into high-energy fuel [35-37]. Relative to the expensive noble catalysts (e.g. Eu, Ru and Pt), the transition element catalysts (e.g. Fe, Mn, Ni and Co) have been reported to be promising candidates for the OER, due to the low price and abundant resources [38-41]. For this purpose, we further investigated the potential electrocatalytic properties of the Co@GCMs samples as candidate catalyst for the OER, with the results shown in Fig. 8. The samples sintered at $800{ }^{\circ} \mathrm{C}$ and $900{ }^{\circ} \mathrm{C}$ are designated as Co@GCMs-1 and Co@GCMs-2, respectively. Fig. 8 A shows the CV curves at $10 \mathrm{mV} \mathrm{s}^{-1}$ of the different samples in $0.1 \mathrm{M} \mathrm{KOH}$ aqueous solution. The onset potentials of Co@GCMs-1 and Co@GCMs-2 are 1.36V and 1.42V respectively, which are much lower than that of starch-derived C (1.57V). The result indicates that the Co@graphited carbon catalysts behave more excellent catalytic properties than the pure amorphous carbon, due to the efficient Co 
component and high conductivity. The linear-sweep voltammograms of the catalysts are depicted in Fig. 8 B. Remarkably, the catalytic current density of Co@GCMs-1 is 6.43 $\mathrm{A} \mathrm{g}^{-1}$ at $1.60 \mathrm{~V}$ potential, which is 1.5 and 6.0 times as high as Co@GCMs-2 $\left(4.08 \mathrm{~A} \mathrm{~g}^{-1}\right)$ and starch-derived $\mathrm{C}$ (1.04 $\left.\mathrm{A} \mathrm{g} \mathrm{g}^{-1}\right)$, respectively. The higher current density of Co@GCMs-1 compared with Co@GCMs-2 suggests that more favorable electrons transport and diffusion of ions happened for the former. Fig. $8 \mathrm{C}$ demonstrates that the Nyquist plots of EIS for the Co@GCMs-1, Co@GCMs-2 and starch-derived C samples. The Co@GCMs samples show near-vertical curves in the low frequency region, indicating they possess more acceptable active area relative to starch-derived $\mathrm{C}$ sample with a quite sloping curve. In the high frequency region (Fig. 8 D), smaller semicircle (smaller charge transfer resistance (CTR)) and a smaller $\mathrm{x}$-intercept (smaller equivalent series resistance (ESR)) were observed for Co@GCMs-1 sample, while much larger semicircle and $\mathrm{x}$-intercept were shown for Co@GCMs-2 sample, suggesting a facilitated electronic/ionic transfer for the Co@GCMs-1 sample, originated from its high-degree graphited structure and unique porous nanofilms morphology.

The electrochemical stability of Co@GCMs-1, Co@GCMs-2 and starch-derived C samples was investigated by means of chronoamperometry at a constant potential of $1.6 \mathrm{~V}$, with the result shown in Fig. 9. At the entire testing time, the oxidation current density of Co@GCMs-1 is obviously higher than those of Co@GCMs-2 and starch-derived C, as shown in Fig. 9A. Compared to the result of Co@GCMs-1, the current density of Co@GCMs-2 decays more quickly and tends to stabilize at $1.27 \mathrm{~A} \mathrm{~g} \mathrm{~g}^{-1}$ at the end of the test. However, the current density on the Co@GCMs-1 decays slowly at the beginning of OER and retain 3.52 A $\mathrm{g}^{-1}$ after $3600 \mathrm{~s}$. The decay of current density indicates the deactivation of the catalyst. It also can be seen that Co@GCMs-1 possesses higher stability for the OER, compared with the Co@GCMs-2 and starch-derived C in the Fig. 9B. After 3500s, Co@GCMs-1maintains a better catalytic activity of around 53.8\%, while Co@GCMs-2 and starch-derived C are 39.8\% and $22.8 \%$, respectively. Therefore, the present results demonstrated that the Co@GCMs-1 sample has a very high catalytic activity and a good stability for the OER.

\section{Conclusion}

Cobalt nanoparticles wrapped in gaphited carbon microspheres (Co@GCMs) have been achieved by the temperature-controlled water-swelling and catalytic graphitization associated strategy from starch microspheres. Structural analysis shows that the Co nanoparticles were wuniformly enwrapped by porous graphited carbon nanofilms. High catalytic activity and good stability for the oxygen evolution reaction (OER) have been demonstrated, when the Co@GCMs was evaluated as a potential catalyst for the oxidation of water. The porous graphited nanofilms grown on Co@GCMs plays a vital role as a catalytic reinforcement, which facilitates the electronic/ionic transfer during the water oxidation into molecular oxygen and thus the OER is accomplished at less positive potentials.

\section{Acknowledgements}

This research was supported by the National Science Foundation of China (U1401246, 51474110, 51474077 and 21443006). 


\section{Notes and References}

[1] H. W. Kroto, J. R. Heath, S. C. O'Brien, R. F. Curl, Smalley, C60: buckminsterfullerene, Nature 318 (1985) 162-163.

[2] S. Iijima, Helical microtubules of graphitic carbon, Nature 354 (1991) 56-58.

[3] D. Jariwala, V. K. Sangwan, L. J. Lauhon, T. J. Marks, M. C. Hersam, Carbon nanomaterials for electronics, optoelectronics, photovoltaics, and sensing, Chem. Soc. Rev. 42 (2013) 2824-2860.

[4] Z. Li, D. Li, Z. Liu, B. Li, C. Ge, Y. Fang, Mesoporous carbon microspheres with high capacitive performances for supercapacitors, Electrochim. Acta 158 (2015) 237-245.

[5] H. Hond and Y. Yamada, Synthesis and stability of mesocarbon microbeads separated from the mesophase pitches, J. Jpn. Petrol. Inst. 16 (1973) 392.

[6] S. I. Lee, S. Mitani, S. H. Yoon, Y. Korai, I. Mochida, Preparation of spherical activated carbon with high electric double-layer capacitance, Carbon 42 (2004) 2332-2334.

[7] Y. S. Wang, C. M. Huang, H. W. Hsieh, Y. F. Lin, C. Y. Lin, J. T. Lee, Effect of temperature on the dissolution of solid electrolyte interface on mesocarbon microbeads electrodes in propylene carbonate-based electrolytes, Electrochim. Acta 142 (2014) 34-42.

[8] J. Zhang, Z. Shi, C. Wang, Effect of temperature on the dissolution of solid electrolyte interface on mesocarbon microbeads electrodes in propylene carbonate-based electrolytes, Electrochim. Acta 125 (2014) 22-28.

[9] M. S. Wu, Y. H. Fu, Electrophoretic self-assembly of expanded mesocarbon microbeads with attached nickel nanoparticles as a high-rate electrode for supercapacitors, Nanoscale 6 (2014) 4195-4203.

[10] L. Zhang, M. Zhang, Y. Wang, Z. Zhang, G. Kan, C. Wang, Z. Zhong, F. Su, Graphitized porous carbon microspheres assembled with carbon black nanoparticles as improved anode materials in Li-ion batteries, J. Mater. Chem. A 2 (2014) 10161-10168.

[11] X. Ma, L. Gan, M. Liu, P. K. Tripathi, Y. Zhao, Z. Xu, D. Zhu, L. Chen, Mesoporous size controllable carbon microspheres and their electrochemical performances for supercapacitor electrodes, J. Mater. Chem. A 2 (2014) 8407-8415.

[12] T. Chen, L. Pan, T. Lu, C. Fu, D. H. C. Chua, Z. Sun, Fast synthesis of carbon microspheres via a microwave-assisted reaction for sodium ion batteries, J. Mater. Chem. A 2 (2014) 1263-1267.

[13] M. M. Titirici, R. J. White, N. Brun, V. L. Budarin, D. S. Su, F. del Monte, J. H. Clark, M. J. MacLachlan, Sustainable carbon materials, Chem. Soc. Rev. 44 (2015) 250-290.

[14] Y. Li, S. Xu, X. Wu, J. Yu, Y. Wang, Y. S. Hu, H. Li, L. Chen, X. Huang, Amorphous monodispersed hard carbon micro-spherules derived from biomass as a high performance negative electrode material for sodium-ion batteries, J. Mater. Chem. A 3 (2015) 71-77.

[15] J. Zhang, J. Xiang, Z. Dong, Y. Liu, Y. Wu, C. Xu and G. Du, Biomass derived activated carbon with 3D connected architecture for rechargeable lithium- sulfur batteries, Electrochim. Acta 116 (2014) 146-151.

[16] M. Wu, P. Ai, M. Tan, B. Jiang, Y. Li, J. Zheng, W. Wu, Z. Li, Q. Zhang, X. He, Synthesis of starch-derived mesoporous carbon for electric double layer capacitor, Chem. Eng. J. 245 (2014) 166-172. 
[17] O. Haibo, L. Cuiyan, H. JianFeng, F. Jie, Synthesis of carbon/carbon composites by hydrothermal carbonization using starch as carbon source, RSC Adv. 4 (2014) 12586-12589.

[18] S. Zhao, C. Wang, M. Chen, Z. Shi, N. Liu, Preparation of carbon spheres from potato starch and its stabilization mechanism, New Carbon Materi. 25 (2010) 438-443.

[19] M. Zheng, H. Zhang, Y. Xiao, H. Dong, Y. Liu, R. Xu, Y. Hu, B. Deng, B. Lei, X. Liu, Large-scale synthesis and enhanced hydrogen storage of monodispersed sulfur-doped carbon microspheres by hydro-sulfur-thermal carbonization of starch, Mater. Lett. 109 (2013) 279-282.

[20] C. Yu, J. S. Qiu, Preparation and magnetic behavior of carbon-encapsulated cobalt and nickel nanoparticles from starch, Chem. Eng. Res. Des. 86 (2008) 904-908.

[21] P. Zheng, T. Liu, J. Zhang, L. Zhang, Y. Liu, J. Huang, S. Guo, Sweet potato-derived carbon nanoparticles as anode for lithium ion battery, RSC Adv. 5 (2015) 40737-40741.

[22] Q. Ruibin, H. Zhongai, Y. Yuying, L. Zhimin, A. Ning, R. Xiaoying, H. Haixiong, W. Hongying, Monodisperse carbon microspheres derived from potato starch for asymmetric supercapacitors, Electrochim. Acta 167 (2015) 303-310.

[23] Q. Y. Li, Y. S. Wu, X. H. Zhang, Y. G. Huang, Q. Wu, H. Q. Wang, Tween 20 surfactant effect on the electrocatalytic performance of porous carbon micro-spheres supported $\mathrm{MnO}_{2}$, RSC Adv. 4 (2014) 52100-52104.

[24] Y. Chen, X. Li, X. Zhou, H. Yao, H. Huang, Y. Mai, L. Zhou, Hollow-tunneled graphitic carbon nanofibers through Ni-diffusion-induced graphitization as high-performance anode materials, Energy Environ. Sci. 7 (2014) 2689-2696.

[25] B. Chang, Y. Guo, Y. Li, H. Yin, S. Zhang, B. Yang, X. Dong, Graphitized hierarchical porous carbon nanospheres: simultaneous activation/graphitization and superior supercapacitance performance, J. Mater. Chem. A 3 (2015) 9565-9577.

[26] G. Yuan, X. Li, Z. Dong, X. Xiong, B. Rand, Z. Cui, Y. Cong, J. Zhang, Y. Li, Z. Zhang, J. Wang, Pitch-based ribbon-shaped carbon-fiber-reinforced one-dimensional carbon/carbon composites with ultrahigh thermal conductivity, Carbon 68 (2014) 413-425.

[27] E. Thompson, A. E. Danks, L. Bourgeois, Z. Schnepp, Iron-catalyzed graphitization of biomass, Green Chem. 17 (2015) 551-556.

[28] H. Lei, Y. Wang, J. Huo, Porous graphitic carbon materials prepared from cornstarch with the assistance of microwave irradiation, Micropor. Mesopor. Mater. 210 (2015) 39-45.

[29] N. Ji, X. Li, C. Qiu, G. Li, Q. Sun, L. Xiong, Effects of heat moisture treatment on the physicochemical properties of starch nanoparticles, Carbohyd. Polym. 117 (2015) 605-609.

[30] J. Goscianska, W. Nowicki, R. Pietrzak, Physicochemical and sorption properties of multi-walled carbon nanotubes decorated with silver nanoparticles, Chem. Eng. J. 250 (2014) 295-302.

[31] Y. Niu, X. Zhang, J. Wu, J. Zhao, X. Yan, Y. Li, Catalytic and enhanced effects of silicon carbide nanoparticles on carbonization and graphitization of polyimide films, RSC Adv. 4 (2014) 42569-42576.

[32] K. Davami, M. Shaygan, N. Kheirabi, J. Zhao, D. A. Kovalenko, M. H. Rümmeli, J. Opitz, G. Cuniberti, J. S. Lee, M. Meyyappan, Synthesis and characterization of carbon nanowalls on different substrates by radio frequency plasma enhanced chemical vapor deposition, Carbon 72 (2014) 372-380. 
[33] Z. Ni, Y. Wang, T. Yu, Z. Shen, Raman spectroscopy and imaging of grapheme, Nano Res. 1 (2008) 273-291.

[34] R. F. Tester, S. J. J Debon, Annealing of starch-a review, Int. J. Biol. Macromol. 27 (2000) 1-12.

[35] X. Long, S. Xiao, Z. Wang, X. Zheng, S. Yang, Co intake mediated formation of ultrathin nanosheets of transition metal $\mathrm{LDH}$ - an advanced electrocatalyst for oxygen evolution reaction, Chem. Commun. 51 (2015) 1120-1123.

[36] E. Fabbri, A. Habereder, K. Waltar, R. Kötz, T. J. Schmidt, Developments and perspectives of oxide-based catalysts for the oxygen evolution reaction, Catal. Sci. Technol. 4 (2014) 3800-3821.

[37] N. Li, W. Y. Xia, J. Wang, Z. L. Liu, Q. Y. Li, S. Z. Chen, C. W. Xu, X. H. Lu, Manganese oxides supported on hydrogenated $\mathrm{TiO} 2$ nanowire array catalysts for the electrochemical oxygen evolution reaction in water electrolysis, J. Mater. Chem. A 3 (2015) 21308-21313.

[38] A. R. Zeradjanin, A. A. Topalov, Q. Van Overmeere, S. Cherevko, X. Chen, E. Ventosa, W. Schuhmann, K. J. Mayrhofer, Rational design of the electrode morphology for oxygen evolution-enhancing the performance for catalytic water oxidation, RSC Adv. 4 (2014) 9579-9587.

[39] A. Sartorel, M. Carraro, F. M. Toma, M. Prato, M. Bonchio, Shaping the beating heart of artificial photosynthesis: oxygenic metal oxide nano-clusters, Energy Environ. Sci. 5 (2012) $5592-5603$.

[40] C. Xu, M. Lu, Y. Zhan, J. Y. Lee, A bifunctional oxygen electrocatalyst from monodisperse $\mathrm{MnCo}_{2} \mathrm{O}_{4}$ nanoparticles on nitrogen enriched carbon nanofibers, RSC Adv. 4 (2014) 25089-25092.

[41] X. Liu, J. Jiang, L. Ai, Non-precious cobalt oxalate microstructures as highly efficient electrocatalysts for oxygen evolution reaction, J. Mater. Chem. A 3 (2015) 9707-9713. 


\title{
Sustainable synthesis of Co NPs@Graphited carbon microspheres as an efficient electrocatalyst for the oxygen-evolution reaction
}

\author{
Hong-Qiang Wang ${ }^{\mathrm{a}}$, Dong-Cai Zhang ${ }^{\mathrm{a}}$, Xiao-Hui Zhang ${ }^{\mathrm{a}}$, Ze-Sheng Li ${ }^{\mathrm{b}}$,, Guan-Hua \\ Yang ${ }^{\mathrm{a}}$, Yong-Sheng $\mathrm{Wu}^{\mathrm{a}}$, Jing-Jing $\mathrm{Ji}^{\mathrm{c}}$ and Qing-Yu Li ${ }^{\mathrm{a}, *}$ \\ a Guangxi Key Laboratory of Low Carbon Energy Materials, School of Chemistry and pharmaceutical Sciences, \\ Guangxi Normal University, Guilin, 541004, China. \\ b Development Center of Technology for Petrochemical Pollution Control and Cleaner Production of Guangdong \\ University, College of Chemical Engineering, Guangdong University of Petrochemical Technology, Maoming, \\ Guangdong, 525000, China. \\ c Ningbo Institute of Materials Technology and Engineering, Chinese Academy of Science, Ningbo, Zhejiang, \\ 315000, China. \\ Correspongding authors \\ E-mail addresses \\ *Qing-Yu Li, lingqingyu62@126.com Fax: +86-0773-5858562 \\ *Ze-Sheng Li, 1zs212@163.com
}

Figure
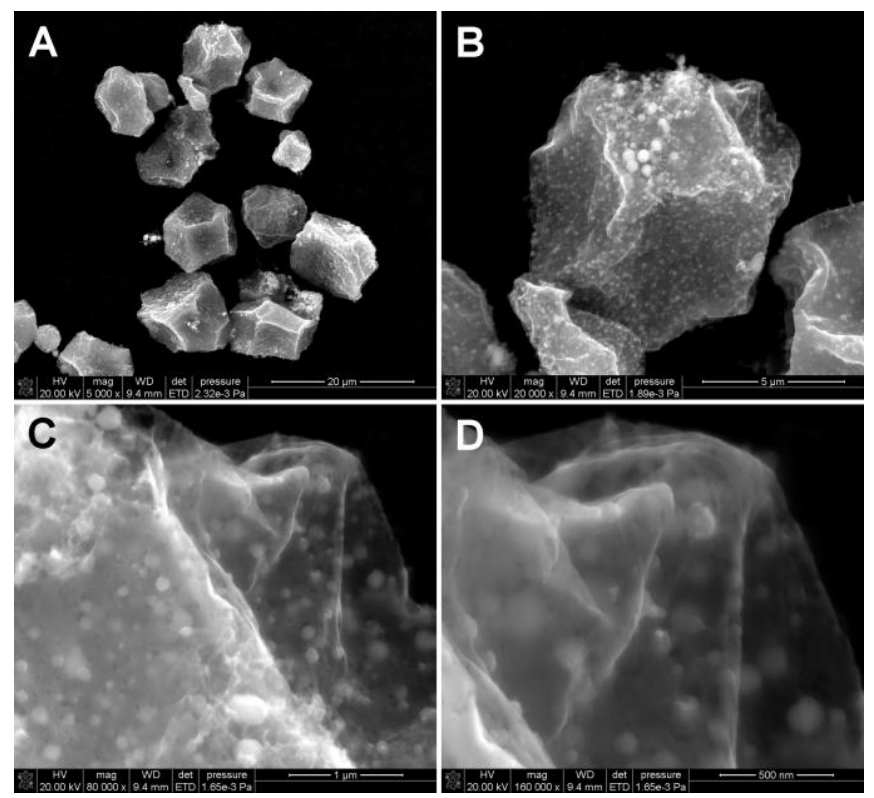

Fig. 1 Typical SEM images of the Co@GCMs sample sintered at $900{ }^{\circ} \mathrm{C}$. 

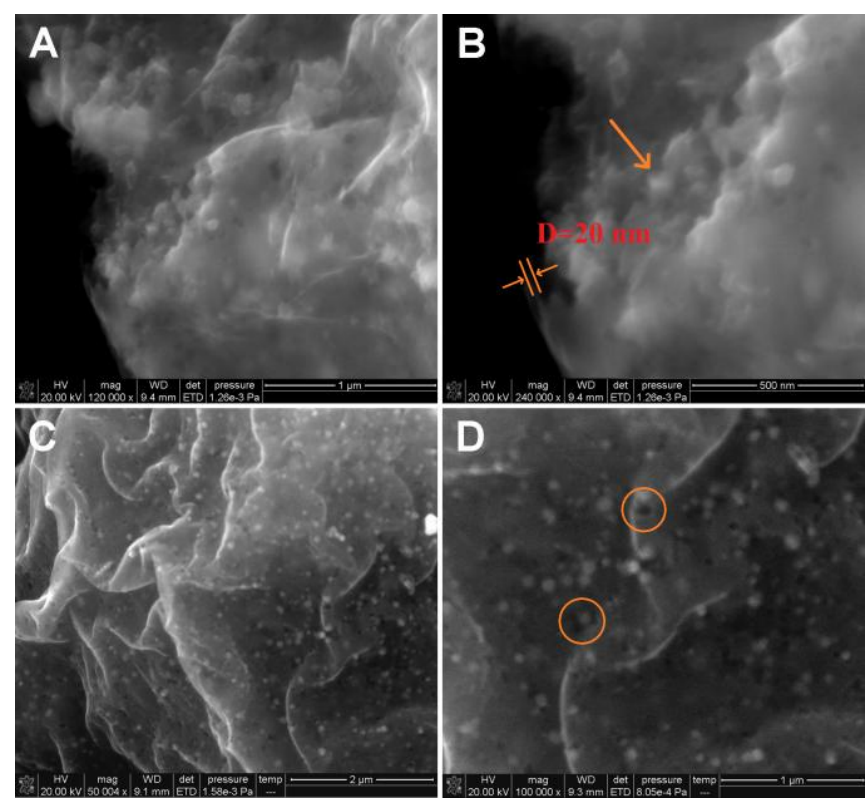

Fig. 2 Internal structures (A,B) and porous structures (C,D) of the Co@GCMs sample sintered at $900{ }^{\circ} \mathrm{C}$.
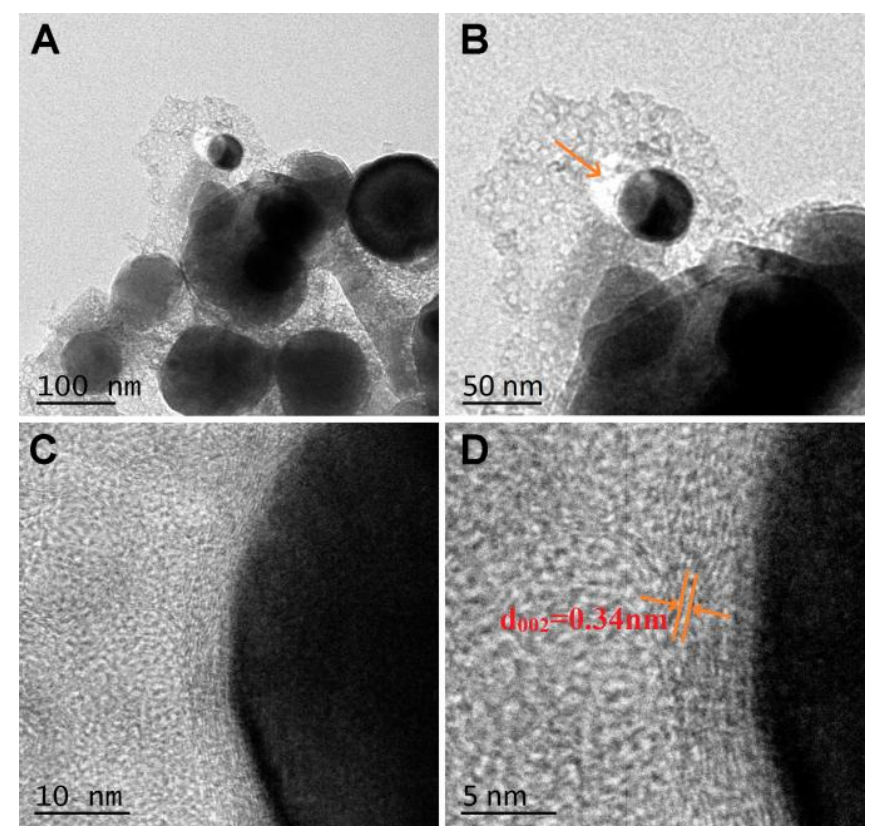

Fig. 3 Typical TEM images of the Co@GCMs sample sintered at $900{ }^{\circ} \mathrm{C}$.
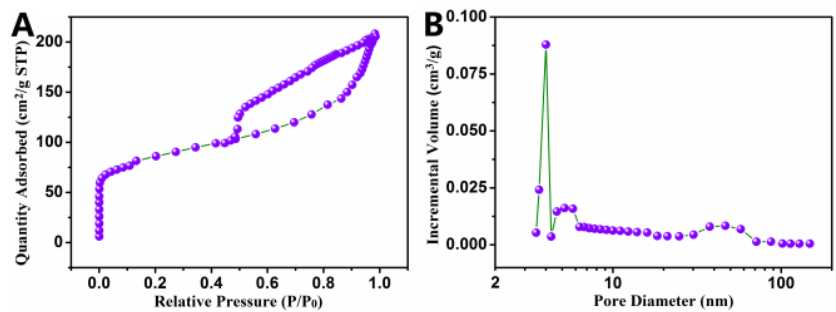

Fig. 4 Nitrogen adsorption-desorption isotherm (A) and pore size distribution (B) of the Co@GCMs sample sintered at $900{ }^{\circ} \mathrm{C}$. 

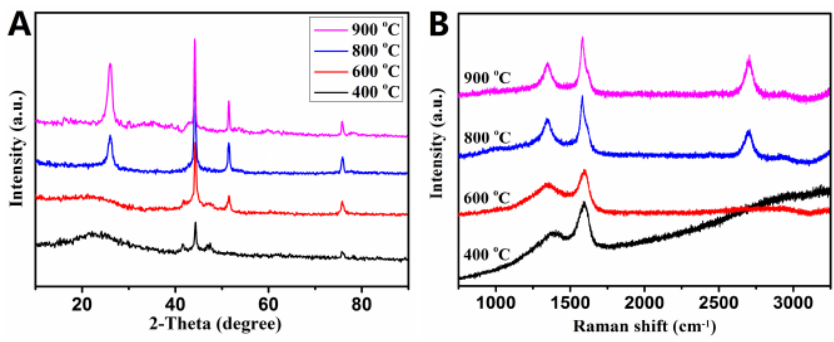

Fig. 5 XRD patterns (A) and Raman spectrums (B) of the samples sintered at different temperatures.

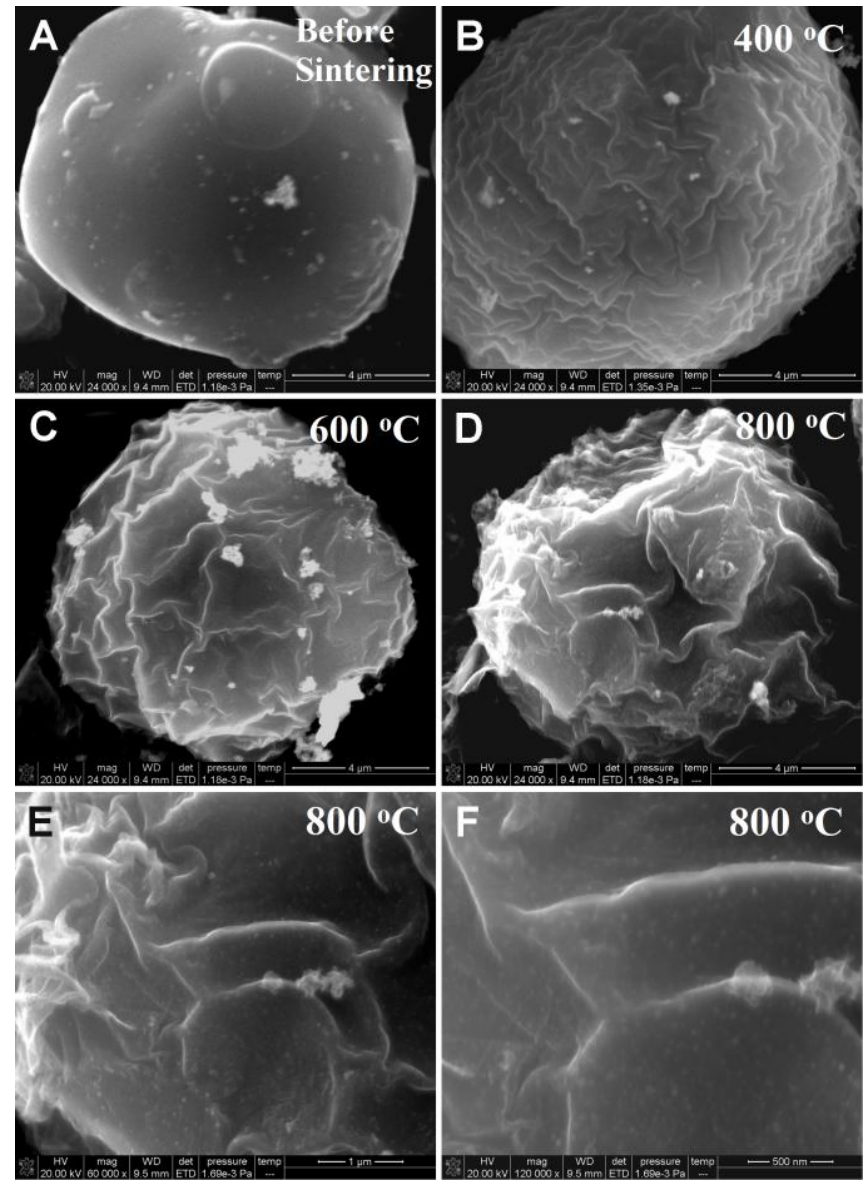

Fig. 6 Comparative SEM images of the sample sintered at different temperatures. A: before sintering, B: $400{ }^{\circ} \mathrm{C}, \mathrm{C}: 600^{\circ} \mathrm{C}, \mathrm{D}: 800^{\circ} \mathrm{C}$, and different magnification of sample at $800{ }^{\circ} \mathrm{C}$ (E, F) 


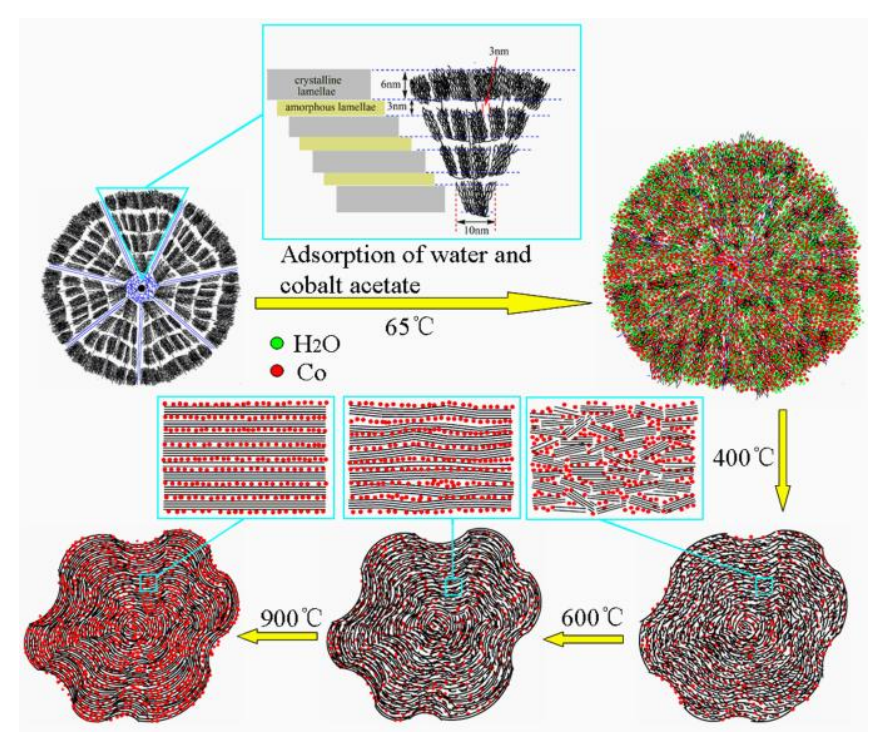

Fig. 7 Schematic illustration for the formation of Co@GCMs from starch microsphere and cobalt acetate.
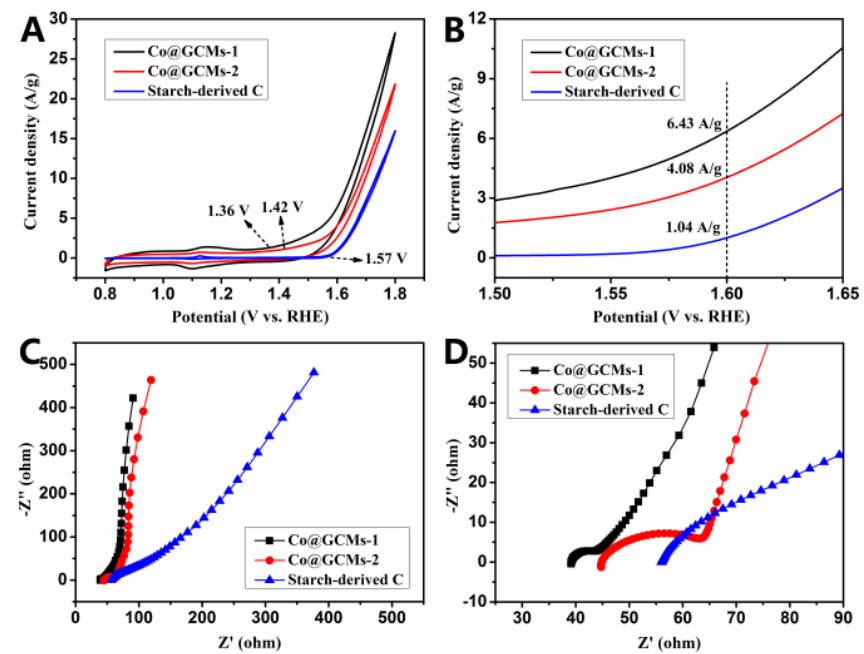

Fig. 8 The CV curves (10 mV/s) (A), linear-sweep voltammograms (B), Nyquist plots (C) and its high frequency region (D) of different samples in $0.1 \mathrm{M} \mathrm{KOH}$.
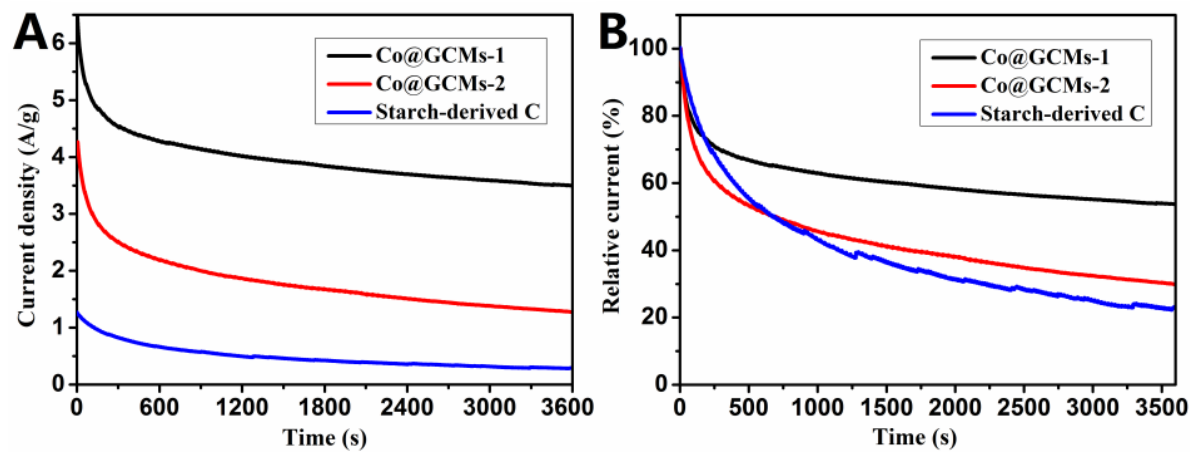

Fig. 9 Chronoamperometric curves of different samples in $0.1 \mathrm{M} \mathrm{KOH}$ with a potential of $1.6 \mathrm{~V}$ : (A) real current $(\mathrm{B})$ relative current. 